\title{
Finite element modelling of the insertion of an electrode array into the cochlear scala tympani with lubricant
}

\author{
Hung Kha ${ }^{1} \quad$ Bernard Chen ${ }^{2}$
}

(Received 14 December 2010; revised 7 December 2011)

\begin{abstract}
Cochlear implants, consisting of electrode arrays for electrically stimulating the auditory nerve fibres, have been developed to restore hearing for profoundly deaf people. The insertion of a straight electrode array into the spiral cochlear scala tympani has unfortunately been found to damage delicate structures within the inner ear, such as the spiral ligament and the basilar membrane. Although a number of studies suggested the use of lubricant for smooth insertion, little attempt has been made to quantify the effect of lubricant on the damage by the electrode array to the cochlear structures. We develop a mathematical model, using the finite element method, to predict the trajectories of the Nucleus standard straight electrode array during insertion into the scala tympani, and associated contact stresses exerted
\end{abstract}

http://journal . austms.org.au/ojs/index.php/ANZIAMJ/article/view/3783 gives this article, (c) Austral. Mathematical Soc. 2011. Published December 13, 2011. ISSN 1446-8735. (Print two pages per sheet of paper.) Copies of this article must not be made otherwise available on the internet; instead link directly to this URL for this article. 
by the array on the cochlear structures. Results from the model show that the use of a lubricant, such as glycerine, reduces the contact stresses exerted by the tip of the array on the spiral ligament from 0.31 to $0.2 \mathrm{MPa}$, whereas the contact stresses on the basilar membrane reduce from $0.075 \mathrm{MPa}$ (without lubricant) to $0.025 \mathrm{MPa}$ (with lubricant). These results suggest that the use of a lubricant is clinically important for minimising the likelihood of damage by the electrode array to the cochlear structures.

\section{Contents}

1 Introduction

C1050

2 Methods

C1052

3 Results and discussion

C1053

4 Conclusions

C1057

References

C1057

\section{Introduction}

Although cochlear implants were successfully developed to restore near-tonormal hearing for deaf people, their implantation has been found to damage the delicate cochlear structures. In each implant system, a microphone (located above the external ear) picks up sound which is sent to a microprocessor and then converted to electrical currents travelling through a straight electrode array (consisting of platinum wires embedded in a Silastic matrix) inserted into the scala tympani (the lower tubular space of the cochlea). The currents stimulate the auditory nerve fibres within the cochlea [2]. As 
Electrode array

Basilar membrane

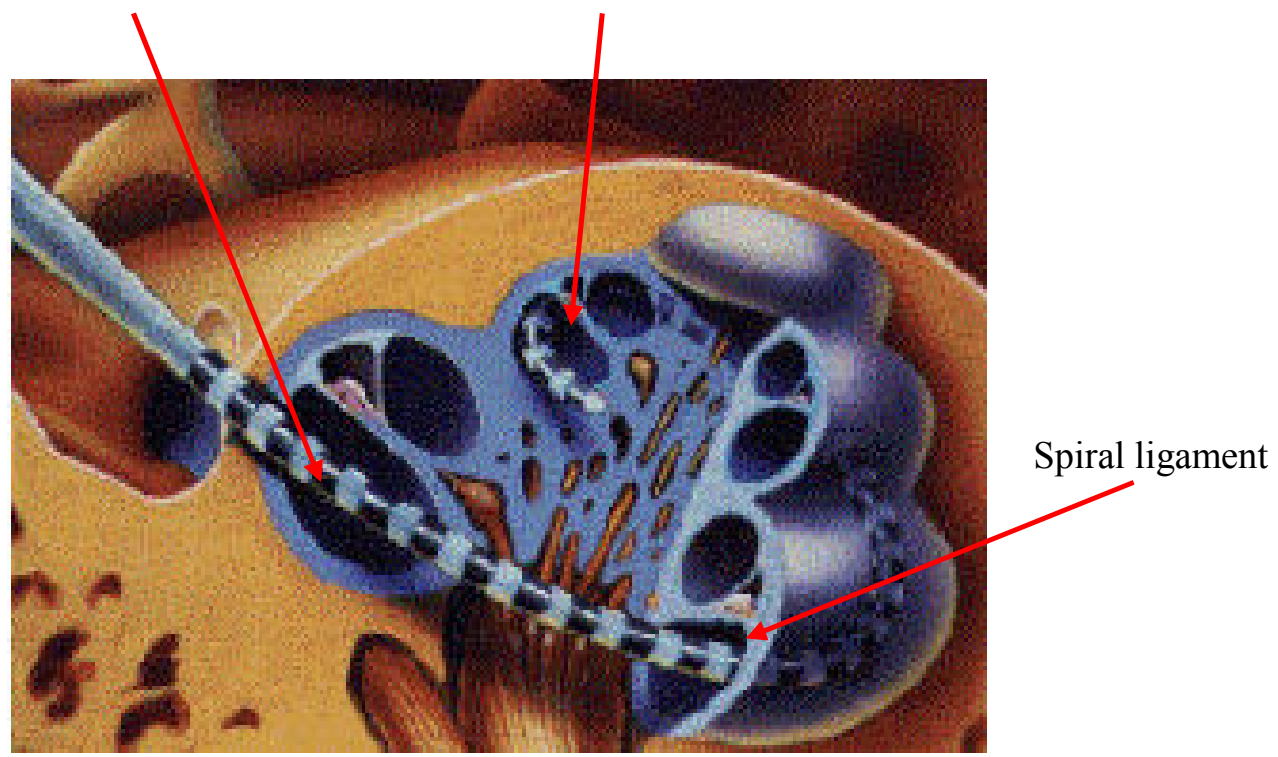

Figure 1: Insertion of an electrode array into the cochlear scala tympani [1].

the cochlea has a spiral shape, the electrode array largely bends and slides against the outer wall of the scala tympani during insertion, which has a great potential for damaging cochlear structures (Figure 1). Results from previous experimental studies, in which electrode arrays were inserted into cochleae harvested from temporal bones, showed that the electrode arrays usually tore the spiral ligament located along the outer wall and eventually pierced the basilar membrane at the upper surface of the scala tympani [10, 12, 13].

A number of studies suggested the use of lubricants for reducing the damage by the electrode array to the cochlear structures. Biocompatible lubricants such as glycerine and hyaluronic acid (Healon) are often used to coat the electrode array with a thin layer to reduce friction between the array and the endosteum lining covering the interior walls of the scala tympani $[7,9,12,6,2,13]$. The electrode arrays in these experiments were reported to be 'smoothly' inserted 
into the scala tympani; however, there has not been any attempt to study quantitatively how the use of these lubricants could reduce the damage to the spiral ligament and the basilar membrane. Although a recent study on cochlear implant insertion by Todd et al. [11] largely involved measurement of insertion forces and coefficients of friction between the electrode array and the scala tympani model, it did not investigate the influence of different lubricants on the damage.

We present a mathematical model using the finite element method (a numerical technique for determining approximate solutions of partial differential equations) to predict the contact stresses exerted by the electrode array on the outer wall and the upper surface of the scala tympani during insertion into the cochlea. The model is used for studying the effect of lubricants such as glycerine on the reduction of damage by the tip of the electrode array to the spiral ligament and the basilar membrane.

\section{Methods}

A mathematical (finite element) model that simulates the insertion of the electrode array into the scala tympani is constructed using the polar coordinates of the cochlear pathway [3] and the cochlear gradient of $15^{\circ}$ [1]. The electrode array model is created using dimensions of the prototypes provided by the manufacturer. The array is $20 \mathrm{~mm}$ in length and tapers from $0.7 \mathrm{~mm}$ at the rear section to $0.45 \mathrm{~mm}$ at the tip. We use 1993 elements for meshing the scala tympani and the electrode array. The Young's modulus of elasticity of the array, which increases from $180 \mathrm{MPa}$ at the rear section to $490 \mathrm{MPa}$ at the front section [5], is incorporated into the model. A surface-to-surface contact algorithm [8] is employed to model the sliding of the electrode array (slave surface) against the scala tympani (master surface). A friction coefficient of 0.19 is used for the case of no lubricant and of 0.12 [4] for the case when glycerine is used as a lubricant. The penetration type for the sliding contact between the electrode array and the scala tympani is selected 
as 'unsymmetrical', in which 'only the penetration of the slave node into the master segments is checked' [8]. Enforced displacements are prescribed at nodes of the basal end of the electrode array, whereas the nodes on the scala tympani are fixed. The finite element code NE/Nastran [8] is used for implementing the non-linear static analysis (Newton-Raphson method) by solving

$$
[\mathrm{K}]\{\Delta \mathrm{D}\}=\{\Delta \mathrm{R}\},
$$

where

$$
\begin{aligned}
K & =\text { global tangent stiffness matrix } \\
\Delta \mathrm{D} & =\text { global incremental displacement vector } \\
\Delta \mathrm{R} & =\text { global incremental load vector. }
\end{aligned}
$$

More than 100 increments and 30 iterations are used to ensure convergence for each linear step (with a convergence criterion for out-of-balance force of between $0.005-0.01 \%$ ). To ensure convergence in each incremental load step, a stiffness scaling factor was chosen between 0.01 and 0.05 . Two sets of experiments validate the finite element model of insertion of the electrode array into the scala tympani. In the first set of experiments, the critical buckling loads of the electrode array are measured and compared with those predicted from the model. In the second set of experiments, the predicted insertion trajectories of the electrode array are compared with those obtained from the fluoroscopy images of insertion of the array into human temporal bones.

\section{Results and discussion}

The predicted results from the finite element model of insertion of the electrode array into the scala tympani agree strongly with those obtained from experiments. First, the critical buckling load of the array predicted by the model is $0.51 \mathrm{~g}$, which is close to experimental values $(0.52 \pm 0.01 \mathrm{~g})$. Second, 


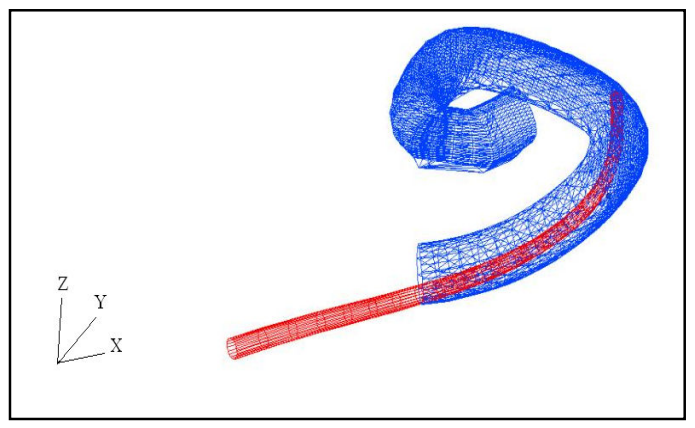

(a)

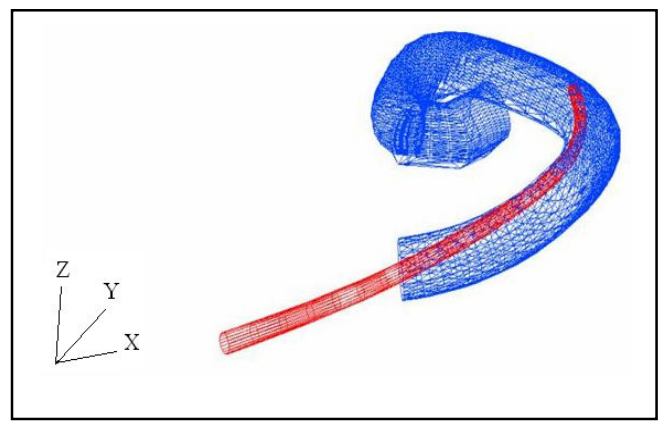

(b)

Figure 2: Predicted bending behaviour of the electrode array: without lubricant (left) and with lubricant (right).

the predicted trajectories of the electrode array during insertion into the scala tympani generally agree with those obtained from experiments of insertion of the electrode array into human temporal bones. Root-mean-square deviations between the predicted coordinates of the four points along the length of the electrode array $(0,4,8$ and $11 \mathrm{~mm}$ from the tip) and the average values of coordinates of these points determined from the experiments at different stages of insertion (the tip at 5, 12, 15 and $22 \mathrm{~mm}$ from the round window) are found to be less than $0.13 \mathrm{~mm}$.

Figure 2 shows bending behaviour of the electrode array during insertion into the scala tympani. The electrode array without lubrication by glycerine (left) is found to experience significantly less bending at the front section (due to more friction between the tip of the array and the endosteum lining), compared to that with the use of lubricant (right). The result is consistent with observations from previous experimental studies in which the electrode array appeared to be more 'smoothly' inserted into the scala tympani and experience less resistance with a lubricant than without a lubricant $[10,7,9,6]$.

The finite element model is used to predict contact stresses exerted by the tip of the electrode array on the outer wall of the scala tympani, which are 


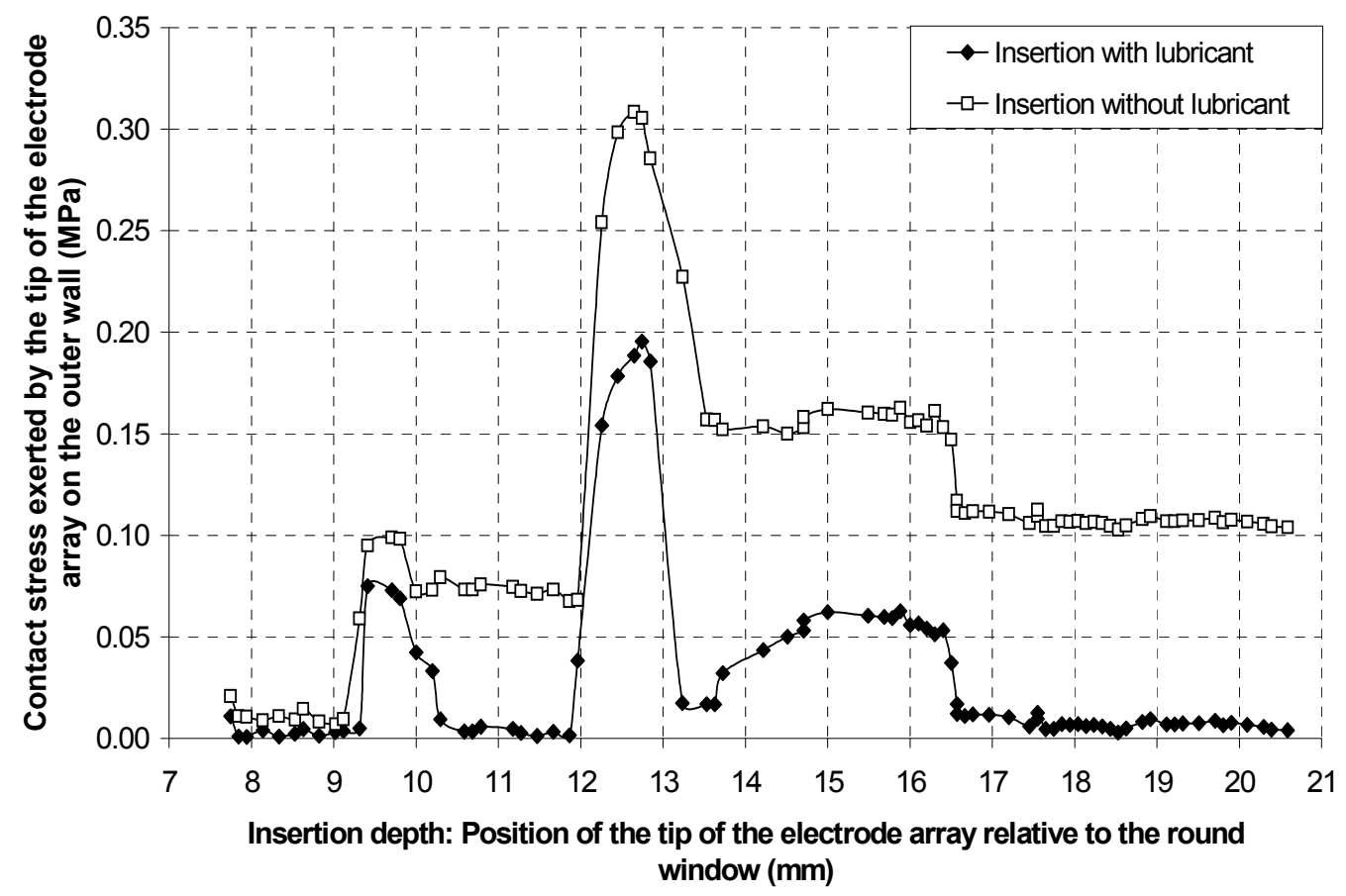

Figure 3: Contact stresses exerted by the tip of the electrode array on the outer wall of the scala tympani in the lubricated and the non-lubricated insertions.

indicative of the damage to the spiral ligament (Figure 3). The stresses in the non-lubricated condition are about 0.1 and $0.31 \mathrm{MPa}$ when the tip is at $9-10 \mathrm{~mm}$ and $12-13 \mathrm{~mm}$ from the round window (the entry for insertion into the scala tympani), respectively. These stresses are higher than those exerted by the tip of the array on the outer wall in the lubricated insertion (about 0.07 and $0.2 \mathrm{MPa}$, respectively). Without lubrication, the tip of the electrode array exerted relatively high contact stresses (up to about $0.17 \mathrm{MPa}$ ) on the outer wall beyond $13 \mathrm{~mm}$ from the round window compared to those in the lubricated insertion (up to about $0.07 \mathrm{MPa}$ ). Therefore, there is a greater risk of damaging the spiral ligament without the use of lubricant. 


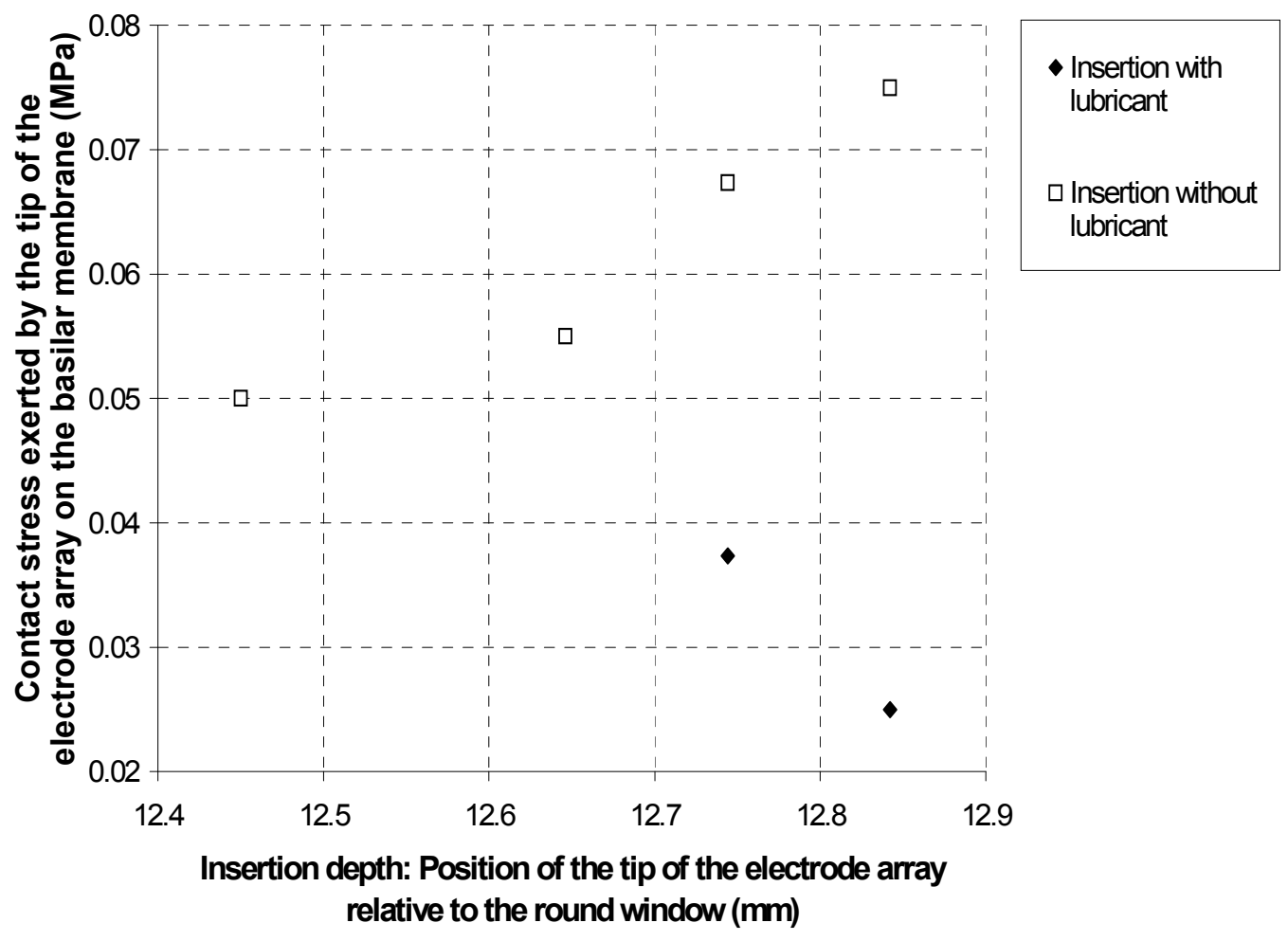

Figure 4: The contact stresses exerted by the tip of the electrode array on the basilar membrane in the non-lubricated and the lubricated insertions.

The model also predicts the effect of a lubricant on the damage by the electrode array to the basilar membrane (Figure 4). The contact stresses exerted by the tip of the array on the upper wall of the scala tympani are relatively high $(0.05-0.075 \mathrm{MPa})$ without lubrication, compared to those with lubrication $(0.02-0.04 \mathrm{MPa})$. The basilar membrane is thus more likely to be damaged by the electrode array in a non-lubricated condition. 


\section{Conclusions}

A mathematical model of insertion of electrode array into the human cochlear scala tympani, using the finite element method, is developed to study the effect of lubricant on the damage by the array to the spiral ligament and the basilar membrane. The use of glycerine is found to reduce significantly the contact stresses exerted by the tip of the array on these cochlear structures. The results confirm quantitatively the importance of using lubricant for reducing damage by the electrode array to the cochlea.

Acknowledgements We thank Professor Graeme Clark and Professor Rhys Jones for their helpful discussion of the research. We are grateful to the Institution of Engineers Australia, the Department of Mechanical Engineering at Monash University and the Vice-Chancellor Office of the Australian National University for financial support.

\section{References}

[1] B. K. Chen, G. M. Clark, and R. Jones. Evaluation of trajectories and contact pressures for the straight nucleus cochlear implant electrode array - a two-dimensional application of finite element analysis. Medical Engineering and Physics, 25:141-147, 2003. doi:10.1016/S1350-4533(02)00150-9. C1051, C1052

[2] G. M. Clark. Cochlear implants-Fundamentals and Applications. Springer-Verlag, New York, 2003. C1050, C1051

[3] L. T. Cohen, J. Xu, S. A. Xu, and G. M. Clark. Improved and simplified methods for specifying positions of the electrode bands of a cochlear implant array. American Journal of Otology, 17:859-865, 1996. C1052 
[4] H. N. Kha and B. K. Chen. Determination of frictional conditions between electrode array and endosteum lining for use in cochlear implant models. Journal of Biomechanics, 39(9):1752-1756, 2006. doi:10.1016/j.jbiomech.2005.04.031. C1052

[5] H. N. Kha, B. K. Chen, G. M. Clark, and R. Jones. Stiffness properties for Nucleus standard straight and Contour electrode arrays. Medical Engineering and Physics, 26:677-685, 2004. doi:10.1016/j.medengphy.2004.05.001. C1052

[6] R. Laszig, G. J. Ridder, and M. Fradis. Intracochlear insertion of electrodes using hyaluronic acid in cochlear implants. Journal of Laryngology Otology, 116:371-372, 2002. doi:10.1258/0022215021910816. C1051, C1054

[7] E. Lehnhardt. In B. Fraysse and O. Deguine, editors, Cochlear implants: new perspectives, volume 48 of Advances in Otorhinolaryngology, pages 62-64. Karger, Basel, 1993. C1051, C1054

[8] Noran Engineering Inc. NE/Nastran Version 8.2 User's Manual, 2002. C1052, C1053

[9] J. J. T. Roland, T. M. Magardino, J. T. Go et al. Effects of glycerine, hyaluronic acid, and hydroxypropyl methylcellulose on the spiral ganglion of the guinea pig cochlea. Annals of Otology, Rhinology and Laryngology Supplement, 166:64-68, 1995. C1051, C1054

[10] R. K. Shepherd, G. M. Clark, B. C. Pyman, and R. L. Webb. Banded intracochlear electrode array: Evaluation of insertion trauma in human temporal bones. Annals of Otology, Rhinology and Laryngology, 94:55-59, 1985. C1051, C1054

[11] C. Todd, F. Naghdy, and M. Svehla. Force application during cochlear implant insertion: An analysis for improvement of surgeon technique. IEEE Transactions on Biomedical Engineering, 54(7):1247-1255, 2007. doi:10.1109/TBME.2007.891937. C1052 
[12] M. Tykocinski, E. Saunders, L. T. Cohen, C. Treaba, R. J. Briggs, P. Gibson, G. M. Clark, and R. S. Cowan. The Contour electrode array: safety study and initial patient trials of a new perimodiolar design. Otology Neurotology, 22:33-41, 2001. C1051

[13] P. Wardrop, D. Whinney, S. J. Rebscher, J. T. J. Roland, W. Luxford, and P. A. Leake. A temporal bone study of insertion trauma and intracochlear position of cochlear implant electrodes. I: comparison of Nucleus banded and Nucleus Contour electrodes. Hearing Research, 203:54-67, 2005. doi:10.1016/j.heares.2004.11.006. C1051

\section{Author addresses}

1. Hung Kha, School of Engineering, Australian National University. mailto: hung. kha@anu.edu.au

2. Bernard Chen, Department of Mechanical and Aerospace Engineering, Monash University.

mailto: bernard. chen@monash. edu 